\title{
A New Uniformly Ultimate Boundedness Criterion for Discrete-Time Nonlinear Systems
}

\author{
Zhishuai Ding ${ }^{1}$, Guifang Cheng ${ }^{1,2}$ \\ ${ }^{1}$ Department of Mathematics, Zhengzhou University, Zhengzhou, China \\ ${ }^{2}$ Physical Engineering College, Zhengzhou University, Zhengzhou, China \\ E-mail: zsding@163.com,gfcheng@zzu.edu.cn \\ Received August 11, 2011; revised September 27, 2011; aceepted October 5, 2011
}

\begin{abstract}
A new type criterion of globally uniformly ultimate boundedness for discrete-time nonlinear systems is introduced. In classical Lyapunov theory about globally uniformly ultimate boundedness, Lyapunov function is assumed to be positive definite and its difference at the every latter moment and the former moment is negative definite. In this paper the condition of difference of Lyapunov function is relaxed. Under the relaxed condition, the result of this paper can be considered as the extension of the classical Lyapunov theory about uniformly ultimate boundedness.
\end{abstract}

Keywords: Uniformly Ultimate Boundedness, Lyapunov Function, Discrete-Time Nonlinear Systems

\section{Introduction}

Discrete-time systems of difference equations have attracted considerable attention. A remarkable book [1] by Agarwal makes the general survey on theory of difference equation and introduces a detailed account of the application of difference equations. Stability analysis is one of the main issues in the area of control systems theory.The classical Lyapunov theory [2] is an important tool to discuss the stability and stabilization problems of dynamical systems. A candidate Lyapunov function is required to satisfy positive definite, and its difference be negative definite or semi-negative definite at the every latter moment and the former moment [3-4]. For discrete-time nonlinear systems, the relation of smooth Lyapunov function and asymptotical stability is presented in paper [5]. In paper [6], new results on the existence of Lyapunov function are presented for discrete-time systems described by difference inclusions. Some new characterizations of uniform global asymptotic stability for nonlinear timevarying discrete-time systems with or without an output-dominant perturbation are proposed on the basis of the detectability for the reduced limiting systems associated with the original system in paper [7]. A new asymptotic stability criterion for nonlinear time-varying differential equations is demonstrated in paper [8] by Aeyels and Peuteman. The Lyapunov function need not be differentiable, and not even be Lipschitz continuous.
Under the relaxed condition, a new asymptotic stability criterion is introduced.

Uniform boundness and uniformly ultimate boundedness is an indispensable part of stability problems [1,2]. In paper [9], Aeyels, Peuteman and Sepulchre transform the problem of uniform boundedness and uniform ultimate boundedness for nonautonomous continuous systems to time-invariant frozen systems and introduced some important results. $\mathrm{Bu}$ and $\mathrm{Mu}$ [10] extend those results and present Lyapunov theorems of uniform boundedness and uniform ultimate boundedness for nonautonomous homogeneous systems. Paper [11] by Cheng, $\mathrm{Mu}$ and Ding discusses the problem of uniformly ultimate boundedness of nonautonomous nonlinear systems with discontinuous right-hand sides and gives some results based on differential inclusions and Filippov solutions. Under arbitrary switching laws, a continuous state feedback control [12] scheme is proposed in order to guarantee uniformly ultimate boundedness of every system response within an arbitrary small neighborhood of the zero state.

In the research area about uniformly ultimate boundedness, the condition of Lyapunov function usually considered is differentiable or Lipschitz continuous and regular. In this paper, based on [8] given by Aeyels and Peuteman, we relax the assumption condition of Lyapunov function. We don't suppose the difference of Lyapunov function at the every latter moment and the former 
moment is negative definite but suppose there exists a finite integer such that Lyapunov function satisfies certain condition. Our object is to provide a new uniformly ultimate boundedness criterion for discrete-time nonlinear systems.

Without loss of generality, $R^{n}$ denotes the n-dimensional Euclidean space, the notation $\|\cdot\|$ is used to denote the Euclidean 2-norm of a vector. $Z$ denotes the set of integers. Continuous function $\alpha(\cdot)$ is said to be a function of class $K$ if, it is strictly increasing and $\alpha(0)=0$. A closed ball $B_{\tau}$ is denoted by

$B_{\tau}=\{x \mid\|x\| \leq \tau\}$.

The rest of the paper is organized as follows. Mathematical preliminary is stated in Section 2. A new type criterion of uniformly ultimate boundedness for nonlinear discrete-time systems is proposed in Section 3. A brief conclusion is provided to summarize the paper in Section 4. Finally, acknowledgements are given in the final section.

\section{Mathematical Preliminary of the Problem}

Consider the systems

$$
x(k+1)=f(x(k), k)
$$

where $x(k) \in R^{n}$ is the state vector at time instant $k$, $k \in Z$. We assume that $f: R^{n} \times Z \rightarrow R^{n}$ satisfies $f(0, k)=0$ for all $k \in Z$ and is globally Lipschitz continuous. Without loss of generality, let $L$ be the Lipschitz constant. Thus the existence and uniqueness of the solution of system (1) is satisfied. $x(k)$ denotes the solution with the initial value $x\left(k_{0}\right)=x_{0}$.

Firstly the definitions of globally uniform boundedness and uniformly ultimate boundedness for discretetime nonlinear systems (1) arepresented.

Definition 2.1. The origin of system (1) is said to be globally uniformly bounded if, for any positive constant $a>0$, there is $b=b(a)>0$ (independent of $k_{0}$ ), such that when $\left\|x\left(k_{0}\right)\right\| \leq a$, there holds

$$
\|x(k)\| \leq b, \forall k \geq k_{0} \text {. }
$$

Definition 2.2. The origin of system (1) is said to be globally uniformly ultimately bounded if, there exist positive constants $r$, for all $\sigma>0$, there is

$K=K(\sigma, r) \geq 0$ (independent of $\left.k_{0}\right)$, such that when $\left\|x\left(k_{0}\right)\right\| \leq \sigma$, there holds

$$
\|x(k)\| \leq r, \forall k \geq k_{0}+K(\sigma, r) .
$$

Lemma 2.3. ([5]) For all $\mu>0$, and choose the closed ball $B_{\mu} \subset R^{n}$. Then for any finite integer $m>0$, there exists $\mu^{\prime}>0$ such that for all $\left(x_{0}, k_{0}\right) \in B_{\mu^{\prime}} \times Z$, there holds

$$
\|x(k)\| \leq \mu, \forall k \in\left[k_{0}, k_{0}+m\right] .
$$

\section{Main Result}

A new type globally uniformly ultimate boundedness criterion for nonlinear discrete-time systems is proposed in this section.

Theorem 3.1. There exists a Lyapunov function $W: R^{n} \times Z \rightarrow R$ which satisfies:

1) there exist two functions $\alpha_{1}, \alpha_{2}$ of class $K$, such that

$$
\alpha_{1}(\|x(k)\|) \leq W(x(k), k) \leq \alpha_{2}(\|x(k)\|) ;
$$

2) for all $\mu>0$, choose $\mu^{\prime}=\mu e^{-L m}$, there exists $0<\tau<\alpha_{2}^{-1}\left(\alpha_{1}\left(\mu^{\prime}\right)\right)$, while for all $\|x\| \geq \tau$, there exists a function $\alpha_{3}$ of class $K$, and a finite integer $m>0$, such that

$$
W(x(k+m), k+m)-W(x(k), k) \leq-\alpha_{3}(\|x(k)\|) .
$$

Then the origin of system (1) is globally uniformly ultimate bounded.

Proof: Choose arbitrary initial value $\left(x_{0}, k_{0}\right) \in B_{\mu^{\prime}} \times Z$, there are only two cases:

(I) $W\left(x_{0}, k_{0}\right) \leq \alpha_{2}(\tau)$; (II) $W\left(x_{0}, k_{0}\right)>\alpha_{2}(\tau)$.

Case I: When (I) holds, then we have

$$
W\left(x\left(k_{0}+m\right), k_{0}+m\right)<W\left(x\left(k_{0}\right), k_{0}\right) \leq \alpha_{2}(\tau) ;
$$

and

$$
W\left(x\left(k_{0}+2 m\right), k_{0}+2 m\right)<W\left(x\left(k_{0}\right), k_{0}\right) \leq \alpha_{2}(\tau) .
$$

By iterative approach, for all $l \in Z_{\geq 0}$, there holds

$$
W\left(x\left(k_{0}+\operatorname{lm}\right), k_{0}+\operatorname{lm}\right)<W\left(x\left(k_{0}\right), k_{0}\right) \leq \alpha_{2}(\tau) .
$$

From (2), we have

$$
\left\|x\left(k_{0}+\operatorname{lm}\right)\right\| \leq \alpha_{1}^{-1} \circ \alpha_{2}\left(\alpha_{2}^{-1}\left(\alpha_{1}\left(\mu^{\prime}\right)\right)\right)=\mu^{\prime} .
$$

Hence for all $k \geq k_{0}$, there exists a constant $k \in Z_{\geq 0}$, such that $k-k_{0}-K m=m$, and

$$
\|x(k)\|=\left\|x\left(k_{0}+(K+1) m\right)\right\| \leq \mu^{\prime} .
$$

By Lemma 2.1, for all $k \geq k_{0}$, there has

$$
\|x(k)\| \leq \mu^{\prime} e^{L m}=\mu .
$$

Case II: When (II) holds, Let

$$
K^{*}=\sup \left\{k \mid W\left(x\left(k_{0}+s m\right), k_{0}+s m\right)>\alpha_{2}(\tau)\right\},
$$

where $0 \leq s \leq k, k \in Z_{\geq 0}$.

We claim $K^{*}<+\infty$.

Contradiction If not, there must hold $K^{*}=+\infty$, and for all $k \in Z_{>0}$, there has

$$
W\left(x\left(k_{0}+k m\right), k_{0}+k m\right)>\alpha_{2}(\tau) .
$$

Then

$$
\alpha_{2}(\tau)<W\left(x\left(k_{0}+k m\right), k_{0}+k m\right)<\alpha_{2}\left(\left\|x\left(k_{0}+k m\right)\right\|\right),
$$


which implies

$$
\left\|x\left(k_{0}+k m\right)\right\|>\tau ; k \in Z_{\geq 0} .
$$

By (4) and iteration, we can get the following inequalities

$$
\begin{aligned}
& W\left(x\left(k_{0}+(k+1) m\right), k_{0}+(k+1) m\right) \\
& -W\left(x\left(k_{0}+k m\right), k_{0}+k m\right) \\
& \leq-\alpha_{3}\left(\left\|x\left(k_{0}+k m\right)\right\|\right) \leq-\alpha_{3}(\tau) ; \\
& W\left(x\left(k_{0}+k m\right), k_{0}+k m\right) \\
& -W\left(x\left(k_{0}+(k-1) m\right), k_{0}+(k-1) m\right) \\
& \leq-\alpha_{3}\left(\left\|x\left(k_{0}+(k-1) m\right)\right\|\right) \leq-\alpha_{3}(\tau) ; \\
& W\left(x\left(k_{0}+T m\right), k_{0}+T m\right)-W\left(x\left(k_{0}\right), k_{0}\right) \\
& <-\alpha_{3}\left(k_{0}\right)<-\alpha_{3}(\tau),
\end{aligned}
$$

then

$$
\begin{aligned}
& W\left(x\left(k_{0}+(k+1) m\right), k_{0}+(k+1) m\right) \\
& <W\left(x\left(k_{0}\right), k_{0}\right)-(k+1) \alpha_{3}(\tau) .
\end{aligned}
$$

When $K \rightarrow+\infty$, clearly $W\left(x\left(k_{0}+(k+1) m\right), k_{0}+(k+1) m\right) \rightarrow-\infty$, which contradicts to the positive definite property of $W(x(k), k)$.

Then there exists an integer $K^{*} \in Z_{\geq 0}$, when $k<K^{*}$, we have $W\left(x\left(k_{0}+k m\right), k_{0}+k m\right)>\alpha_{2}(\tau)$; when $k<K^{*}$, we have

$$
W\left(x\left(k_{0}+k m\right), k_{0}+k m\right) \leq \alpha_{2}(\tau) .
$$

\section{Consequently}

$$
\left\|x\left(k_{0}+k m\right)\right\| \leq \alpha_{1}^{-1} \circ \alpha_{2}\left(\alpha_{2}^{-1}\left(\alpha_{1}\left(\mu^{\prime}\right)\right)\right)=\mu^{\prime} .
$$
has

Furthermore, we can estimate $K^{*}$. By iteration, there

$$
\begin{aligned}
& W\left(x\left(k_{0}+\left(K^{*}-1\right) m\right), k_{0}+\left(K^{*}-1\right) m\right) \\
& <W\left(x\left(k_{0}\right), k_{0}\right)-\left(K^{*}-1\right) \alpha_{3}(\tau)
\end{aligned}
$$

i.e.

$$
\begin{aligned}
& K^{*} \\
& <\frac{W\left(x\left(k_{0}\right), k_{0}\right)-W\left(x\left(k_{0}+\left(K^{*}-1\right) m\right), k_{0}+\left(K^{*}-1\right) m\right)}{\alpha_{3}(\tau)}+1 \\
& <\frac{\left.\alpha_{2}(\sigma)-\alpha_{2}(\tau)\right)}{\alpha_{3}(\tau)}+1 .
\end{aligned}
$$

Then choose

$$
K^{*}(\sigma, \tau)=\frac{\left.\alpha_{2}(\sigma)-\alpha_{2}(\tau)\right)}{\alpha_{3}(\tau)}+1=K^{*}\left(\sigma, \mu^{\prime}\right)=K^{*}(\sigma, \mu)
$$

where $K^{*}(\sigma, \mu)$ is independent of $t_{0}$.

Therefore

$$
\left\|x\left(k_{0}+k T\right)\right\| \leq \alpha_{1}^{-1} \circ \alpha_{2}\left(\alpha_{2}^{-1}\left(\alpha_{1}\left(\mu^{\prime}\right)\right)\right)=\mu^{\prime} .
$$

So in view of Lemma 2, for all $k>k_{0}+K^{*}(\sigma, \mu)$,

$$
\|x(k)\| \leq \mu^{\prime} e^{L m}=\mu \text {. }
$$

The proof is completed.

\section{Conclusions}

We conclude with a brief discussion. In this paper, an extensive Lyapunov theorem of uniform ultimately boundedness is presented. In the classical Lyapunov theory about uniform ultimately boundedness, the difference of Lypunov function at the every latter moment and the former moment is negative definite. Here only need to exist an integer $m$ such that the condition 2) of Theorem 3.1 is satisfied. When $m=1$, the condition 2) becomes the condition in classical Lyapunov theory. Thus theorem 3.1 is less restrictive than that in the classical Lyapunov theory.

\section{Acknowledgements}

This work is supported by the Natural Science Foundation of China under the contracts no. 60874006 and no. 10826078 .

\section{References}

[1] R. P. Agarwal, "Difference Equations and Inequalities: Theory, Methods and Applications,” Marcel Dekker, New York, 1992.

[2] H. K. Khalil, “Nonlinear Systems,” 3rd Edition, Prentice Hall, New York, 2002.

[3] E. D. Sontag, "Comments on Integral Variants of Input-to-State Stability,” Systems and Control Letter, Vol. 34, No. 1, 1998, pp. 93-100. doi:10.1016/S0167-6911(98)00003-6

[4] D. Nesic, A. R. Teel, and E. D. Sontag "Formulas Relating $K L$ Stability Estimates of Discrete-Time and Sampled-Data Nonlinear Systems," Systems and Control Letter, Vol. 38, No. 1, 1999, pp. 49-60. doi:10.1016/S0167-6911(99)00046-8

[5] Z. P. Jiang and Y.Wang, "A converse Lyapunov Theorem for Discrete-Time Systems with Disturbances,” Systems and Control Letter, Vol. 45, No. 1, 2002, pp. 49-58. doi:10.1016/S0167-6911(01)00164-5

[6] C. M. Kellett and A. R. Teel, "Smooth Lyapunov Functions and Robustness of Stability for Difference Inclusions," Systems and Control Letter, Vol. 52, No. 5, 2004, pp. 395-405. doi:10.1016/j.sysconle.2004.02.015

[7] T. C. Lee and Z. P. Jiang, “On Uniform Global Asymptotic Stability of Nonlinear Discrete-Time Systems with 
Applications,” IEEE Transactions on Automatic Control, Vol. 51, No. 10, 2006, pp. 1644-1660.

doi:10.1109/TAC.2006.882770

[8] D. Ayels and J. Peuteman, “A New Asymptotic Stability Criterion for Nonlinear Time-Variant Differential Equation," IEEE Transactions on Automatic Control, Vol. 43, No. 7, 1998, pp. 968-971. doi:10.1109/9.701102

[9] J. Peuteman, D. Aeyels and R. Sepulchre, "Boundededness Properties for Timevarying Nonlinear Systems," SIAM Journal on Control and Optimization, Vol. 39, No. 5, 2000, pp. 1408-1422. doi:10.1137/S0363012999361652

[10] C. X. Bu and X. W. Mu, “A Note for Uniform Bounded- ness of a Class of Timevarying Nonlinear Systems,” Mathematics in Practice and Theory, Vol. 34, No. 6, 2004, pp. 138-142.

[11] G. F. Cheng, X. W. Mu and Z. S. Ding, "Uniformly Ultimate Boundedness for a Class of Discontinuous Nonautonomous Systems," ACTA Mathematicae Applicatae Sinica, Vol. 30, No. 4, 2007, pp. 675-681.

[12] L. G. Zhang, Y. Z. Chen, Z. G. Sun, N. E. Mastorakis and A. Y. Alexsandrov, "Uniformly Ultimate Boundedness Control for Switched Linear Systems with Parameter Uncertainties," Proceedings of 2008 American Control Conference, Seattle, 11-13 June 2008, pp. 3971-3974. doi:10.1109/ACC.2008.4587113 